\title{
Emergencias psiquiátricas en niños y adolescentes: Relación entre características clínicas y demográficas
}

\section{Psychiatric emergencies in children and adolescents: Relationship between clinical and demographic characteristics.}

\author{
Miriam Osorio-Martínez ${ }^{1, a}$, Franklin Peralta-Carmelino 2,b , Oscar Chambergo-Romero 3,b \\ ${ }^{1}$ Servicio de Emergencia, Hospital Hermilio Valdizán, MINSA, Lima, Perú. \\ ${ }^{2}$ Facultad de Medicina Universidad Ricardo Palma, Lima, Perú. \\ ${ }^{3}$ Facultad de Medicina Universidad Nacional Mayor de San Marcos, Lima, Perú.

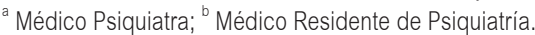

\section{Correspondencia:}

Miriam L. Osorio-Martínez. ml_osorio@hotmail.com

Carretera Central km $3.5 \mathrm{~s} / \mathrm{n}$, Hospital Hermilio Valdizán, DSMAG, Teléfono: 4942410 anexo 232-222.

Recibido: 2 enero 2017

Aceptado: 8 marzo 2017

El siguiente material no ha sido publicado ni remitido a ninguna revista biomédica.

Se obtuvo el consentimiento de la institución para realizar el estudio. Se obtuvo la aprobación del comité de ética.

Conflictos de interés: Ninguno.

Fuente de financiamiento: Propio.

Citar como: Osorio-Martínez M, Peralta-Carmelino F, ChambergoRomero O. Emergencias psiquiátricas en niños y adolescentes: Relación entre características clínicas y demográficas. An Fac med. 2017;78(2): 132-138.

DOI: http://dx.doi.org/10.15381/ anales.v78i2.13185.

\section{An Fac med. 2017;78(2):132-138 / http://dx.doi.org/10.15381/anales.v78i2.13185}

\section{Resumen}

Introducción. Actualmente, la presentación de cuadros agudos de trastornos psiquiátricos es habitual en niños y adolescentes. Objetivo. Determinar la relación entre variables clínicas y demográficas de niños y adolescentes atendidos por emergencia psiquiátrica. Diseño. Estudio cuantitativo, observacional, retrospectivo y analítico. Lugar. Hospital Hermilio Valdizán, Lima, Perú. Participantes. Pacientes menores de 18 años ( $\mathrm{N}=285)$, de ambos sexos, atendidos durante el período setiembre 2014 - setiembre 2015. Intervenciones. Se recopiló información de historias clínicas, clasificándolas en variables clínicas y demográficas. El análisis univariado se realizó por distribución de frecuencias y el bivariado por pruebas chi cuadrado y exacta de Fisher. Resultados. El 13\% de emergencias psiquiátricas fueron pediátricas. Fue más frecuente en el sexo femenino (60\%), grupo etario de 12 a 17 años (93,3\%) y procedencia Lima Este (73\%). La edad media fue 14,8 ( $D E=2,1)$ años. Los motivos de emergencia más frecuentes fueron heteroagresividad $(28,4 \%)$, alucinaciones $(13,7 \%)$, irritabilidad $(11,9 \%)$ y autoagresividad $(10,9 \%)$. La ideación suicida $(9,1 \%)$ estuvo asociada al sexo femenino OR 3,05 (IC-95\%: 1,12-8,35; $\mathrm{p}=0,023)$. Las categorías diagnósticas más frecuentes fueron F20-F29 (31,6\%), F30-F39 (25,6\%) y F60-F69 (18,6\%). El F30-F39 OR 2,31 (IC95\%: 1,28 a 4,16; $p=0,005)$ y F60-F69 OR 2,11 (IC95\%: 1,09 a 4,10; $p=0,025)$ estuvieron asociados al sexo femenino. El $49 \%$ de los pacientes necesitó internamiento. Conclusiones. Existió aumento en prevalencia y menor edad de presentación al comparar con años previos. La heteroagresividad fue el motivo más frecuente de consulta. El sexo femenino tuvo mayor riesgo de Ideación suicida, trastornos del ánimo y personalidad. Se sugiere la elaboración de protocolos estandarizados y manejo especializado.

Palabras clave: Urgencias Médicas; Adolescente; Niño; Suicidio; Trastornos Mentales.

\section{Abstract}

Introduction: Nowadays the presentation of acute psychiatric disorders is common in children and adolescents. Objective: To find the relationship between clinical and demographic variables of children and adolescents seen in the psychiatric emergency room. Design: Quantitative, observational, retrospective and analytical study. Setting: Hermilio Valdizán Hospital, Lima, Peru. Participants: Patients under 18 years ( $N=$ 285), both sexes, seen from September 2014 - September 2015. Interventions: Information was collected from medical records and classified into clinical and demographic variables. Univariate analysis was performed by frequency distribution and bivariate analysis by Chi square and Fisher exact tests. Results: $13 \%$ of all psychiatric emergencies were pediatric. These were more frequent in the female sex (60\%), in the 12 to 17 age group (93.3\%) and in the Lima Este origin group (73\%). Mean age was 14.8 years (SD = 2.1). Aggressiveness (28.4\%), hallucinations $(13.7 \%)$, irritability $(11.9 \%)$ and self-aggressiveness $(10.9 \%)$ were the most frequent chief complaints. Suicidal ideation (9.1\%) was associated with the female group OR 3.05 (IC-95\%: 1.128.35; $p=0.023)$. F20-F29 (31.6\%), F30-F39 (25.6\%), and F60-F69 (18.6\%) were the most frequent diagnostic categories. F30-F39 OR 2.31 (IC-95\%: 1.28-4.16; $p=0.005$ ) and F60-F69 OR 2.11 (IC-95\%: 1.09-4.10; $p=$ 0.025 ) were associated with the female group. $49 \%$ of patients required hospitalization. Conclusions: There were a higher incidence and a lower age of presentation compared with previous years. Aggressiveness was the most frequent chief complaint. The female group had a higher risk of suicidal ideation, mood and personality disorders. The development of standardized protocols and specialized management is suggested. Keywords: Emergencies; Adolescent; Child; Suicide; Mental disorders. 


\section{INTRODUCCIÓN}

La infancia y adolescencia presentan múltiples factores de riesgo para el inicio de los trastornos mentales, ocasionando cuadros agudos que vulneran la integridad del paciente y su entorno. Esta población es vulnerable a la violencia, en especial la adolescencia, con un riesgo de exposición del 50\% a 60\% ${ }^{(1)}$. Estudios realizados en Inglaterra informaron que, en adolescentes atendidos por autoagresión, el $27,3 \%$ volvió a presentar un nuevo episodio en los siguientes 1 a 7 años. Al menos 9,9/1 000 mueren en los próximos 1 a 10 años (2).

El inicio de algunos trastornos mentales es frecuente en estas etapas. Sin embargo, el tratamiento no se administra hasta varios años después ${ }^{(3)}$. Otros autores confirmaron que los trastornos mentales en adultos se habían iniciado con mayor frecuencia desde la etapa de adolescencia $^{(4,5)}$.

Los trastornos psiquiátricos tempranos tienen efectos negativos en múltiples dominios del desarrollo infantil ${ }^{(6)}$ y están asociados al fracaso escolar, embarazos no planificados, crimen y desórdenes psiquiátricos en la adultez ${ }^{(7)}$. A pesar de este escenario, se estima que solo un pequeño porcentaje toma contacto con un servicio de salud mental.

Así, la presentación de emergencias psiquiátricas en niños y adolescentes es cada vez más frecuente. En Italia se informó un ascenso de hasta 38\% del 2001 al $2004^{(8)}$. En un estudio realizado en Inglaterra, el $4 \%$ de menores entre 10 y 19 años tiene al menos alguna atención por emergencia, siendo la tercera parte de ellos por violencia ${ }^{(9)}$ En Perú, un estudio realizado en el Hospital Hermilio Valdizán (2006), reveló que las emergencias en adolescentes incrementaron entre los años 2003 y 2005, siendo la edad más frecuente los 17 años ${ }^{(10)}$. Es escasa la información respecto a la situación en los últimos años.

El objetivo principal de esta investigación fue determinar el perfil clínico y demográfico actual de la población pediátrica atendida por emergencia psiquiátrica.

\section{MÉTODOS}

El presente estudio fue de tipo cuantitativo, observacional, retrospectivo y analítico. La población estuvo conformada por todos los pacientes menores de 18 años, de ambos sexos, atendidos en el Servicio de Emergencia del Hospital Hermilio Valdizán (HHV) durante el período setiembre 2014 a setiembre 2015. Se revisaron todas las historias clínicas de este periodo, excluyendo aquellas que no contaban con un registro completo. Se recopiló información de variables clínicas (motivo de consulta, diagnóstico $\mathrm{CIE}-10$, tratamiento, horario de atención y destino de derivación) y demográficas (procedencia, grupo etario y sexo). La información revisada y recogida en las historias clínicas se manejó de manera confidencial. Este trabajo fue evaluado y aprobado por el Comité de Ética del HHV.

El análisis estadístico se realizó por medio del programa estadístico SPSS versión 21,0, y el análisis univariado por medio de tablas de distribución de frecuencias para todas las variables de estudio. El análisis bivariado usó tablas de contingencia comparando las variables de estudio de acuerdo al sexo y grupo etario por medio de las pruebas chi cuadrado y exacta de Fisher; esta última fue aplicada cuando se encontró valores esperados menores a cinco. Además, se obtuvo el OR con un intervalo de confianza al 95\% para estimar el nivel de asociación entre las variables, considerando para ello como categorías de interés (o riesgo) el sexo mujer y el grupo etario de 12 a 17 años. Estas pruebas fueron trabajadas a un nivel de significancia de $5 \%$.

\section{RESULTADOS}

Se atendió un total de 2187 pacientes con emergencias psiquiátricas durante el período setiembre 2014 a setiembre 2015, de los cuales 285 (13\%) fueron menores de 18 años. La población estuvo compuesta por 171 mujeres (60\%) y 114 varones (40\%) entre 5 a 17 años de edad, con una edad media de 14,8 (DE=2,1). Las atenciones fueron más frecuentes en el grupo etario de 12 a 17 años (266; $93,3 \%)$ y en aquellos procedentes de Lima Este (208; 73\%).

Losmotivos deconsultamásfrecuentes fueron heteroagresividad, alucinaciones, irritabilidad y autoagresividad, en 81 $(28,4 \%), 39(13,7 \%), 34(11,9 \%)$ y 31 $(10,9 \%)$ pacientes, respectivamente (tabla 1). El riesgo suicida se presentó tanto como intento suicida en $16(5,6 \%)$ pacientes e ideación suicida en 26 (9,1\%) pacientes, presentando este último grupo asociación con el sexo femenino, con OR 3,05 (IC95\%: 1,12 a 8,35; $p=$ $0,023)$. Es importante indicar que, en muchos casos, pudieron presentar más de un motivo de consulta (tabla 2).

Tabla 1. Motivo de emergencia psiquiátrica en niños y adolescentes.

\begin{tabular}{lcc}
\multicolumn{1}{c}{ Síntomas * } & $\mathrm{n}$ & $\% \dagger$ \\
\hline Heteroagresividad & 81 & 28,4 \\
Alucinaciones & 39 & 13,7 \\
Irritabilidad & 34 & 11,9 \\
Autoagresividad & 31 & 10,9 \\
Ideación suicida & 26 & 9,1 \\
Ansiedad & 23 & 8,1 \\
Delusiones & 22 & 7,7 \\
Ánimo depresivo & 21 & 7,4 \\
Agitación psicomotriz & 18 & 6,3 \\
Intento suicida & 16 & 5,6 \\
Conducta desorganizada & 14 & 4,9 \\
Otros & 52 & 19,1 \\
\hline
\end{tabular}

${ }^{*}$ Porcentaje en base al total de atenciones por emergencia pediátrica.

†Pacientes presentaron más de un motivo de emergencia.

Los grupos de trastornos asociados más frecuentes fueron: esquizofrenia, trastornos esquizotípico y de ideas delirantes [F20-F29] en 90 (31,6\%) pacientes, trastornos del humor (afectivos) [F30-F39] en 73 (25,6\%) pacientes y trastornos de la personalidad y del comportamiento [F60-F69] en 53 (18,6\%) pacientes. Los diagnósticos $\mathrm{CIE}-10$ más frecuentes en cada grupo fueron: F20.0, F32.0 y F60.3 con 58 (64,4\%), 23 (31,5\%) y $38(71,7 \%)$ pacientes, respectivamente. Aquellos que presentaron una asociación negativa, estadísticamente significativa, con el sexo femenino fueron los grupos trastornos mentales y del comporta- 
Tabla 2. Motivo de consulta de emergencias psiquiátricas en niños y adolescentes según sexo y grupo etario.

\begin{tabular}{|c|c|c|c|c|c|c|c|c|c|c|c|c|c|c|c|c|}
\hline \multirow[b]{3}{*}{ Motivo de consulta§ } & \multicolumn{8}{|c|}{ Sexo } & \multicolumn{8}{|c|}{ Grupo etario } \\
\hline & \multicolumn{2}{|c|}{$\begin{array}{c}\text { Varón } \\
(\mathrm{n}=114)\end{array}$} & \multicolumn{2}{|c|}{$\begin{array}{c}\text { Mujer } \\
(n=171)\end{array}$} & \multicolumn{4}{|c|}{ IC-95\% } & \multicolumn{2}{|c|}{$\begin{array}{c}5 \text { a } 11 \text { años } \\
(n=19)\end{array}$} & \multicolumn{2}{|c|}{$\begin{array}{c}12 \text { a } 17 \text { años } \\
(\mathrm{n}=266)\end{array}$} & \multicolumn{4}{|c|}{ IC95\% } \\
\hline & $\mathrm{n}$ & $\%$ & $\mathrm{n}$ & $\%$ & $\mathrm{OR}^{*}$ & LI & LS & Valor $p$ & $\mathrm{n}$ & $\%$ & $\mathrm{n}$ & $\%$ & ORt & $\mathrm{LI}$ & LS & Valor $\mathrm{p}$ \\
\hline Agitación psicomotriz & 7 & 6,1 & 11 & 6,4 & 1,05 & 0,40 & 2,80 & 0,921 & 3 & 15,8 & 15 & 5,6 & 0,32 & 0,08 & 1,22 & 0,108 \\
\hline Alucinaciones & 16 & 14,0 & 23 & 13,5 & 0,95 & 0,48 & 1,89 & 0,888 & 4 & 21,1 & 35 & 13,2 & 0,57 & 1,18 & 1,81 & 0,308 \\
\hline Ánimo depresivo & 14 & 3,5 & 17 & 9,9 & 3,03 & 0,99 & 9,27 & 0,062 & 3 & 15,8 & 18 & 6,8 & 0,39 & 0,10 & 1,45 & 0,155 \\
\hline Autoagresividad & 10 & 8,8 & 21 & 12,3 & 1,46 & 0,66 & 3,22 & 0,351 & 3 & 15,8 & 28 & 10,5 & 0,63 & 0,17 & 2,29 & 0,445 \\
\hline Ansiedad & 11 & 9,6 & 12 & 7,0 & 0,71 & 0,30 & 1,66 & 0,424 & 1 & 5,3 & 22 & 8,3 & 1,62 & 0,21 & 12,74 & 1 \\
\hline Conducta desorganizada & 6 & 5,3 & 8 & 4,7 & 0,88 & 0,30 & 2,62 & 0,823 & 0 & 0,0 & 14 & 5,3 & NC & & & 0,609 \\
\hline Delusiones & 11 & 9,6 & 11 & 6,4 & 0,64 & 0,27 & 1,54 & 0,319 & 2 & 10,5 & 20 & 7,5 & 0,69 & 0,15 & 3,21 & 0,648 \\
\hline Heteroagresividad & 38 & 33,3 & 43 & 25,1 & 0,67 & 0,40 & 1,13 & 0,133 & 5 & 26,3 & 76 & 28,6 & 1,12 & 0,39 & 3,22 & 0,833 \\
\hline Irritabilidad & 14 & 12,3 & 20 & 11,7 & 0,95 & 0,46 & 1,96 & 0,881 & 2 & 10,5 & 32 & 12,0 & 1,62 & 0,26 & 5,27 & 1 \\
\hline Intento suicida & 5 & 4,4 & 11 & 6,4 & 1,50 & 0,51 & 4,44 & 0,462 & 1 & 5,3 & 15 & 5,6 & 1,08 & 0,13 & 8,61 & 1 \\
\hline Impulsividad & 2 & 1,8 & 10 & 5,8 & 3,48 & 0,75 & 16,18 & 0,132 & 0 & 0,0 & 12 & 4,5 & $\mathrm{NC}$ & & & 1 \\
\hline Ideación suicida & 5 & 4,4 & 21 & 12,3 & 3,05 & 1,12 & 8,35 & 0,023 & 0 & 0,0 & 26 & 9,8 & $\mathrm{NC}$ & & & 0,234 \\
\hline Otros & 20 & 17,5 & 20 & 11,7 & 0,62 & 0,32 & 1,22 & 0,164 & 0 & 0,0 & 40 & 15,0 & $\mathrm{NC}$ & & & 0,086 \\
\hline
\end{tabular}

${ }^{*}$ Condición de riesgo: sexo mujer. † Condición de riesgo: grupo etario 12 a 17 años. § Más de una alternativa posible.

miento por sustancias psicoactivas [F- mente significativa con el sexo femenino 10-F19], con OR 0,15 (IC95\%: 0,04 a 0,55; fueron: trastornos del humor (afectivos) $p=0,001$ ), y esquizofrenia y trastornos es- [F30-F39] OR 2,31 (IC95\%: 1,28 a 4,16; quizotípico y de ideas delirantes [F20-29] $\quad \mathrm{p}=0,005)$ y trastornos de la personalidad OR 0,45 (IC95\%: 0,27 a 0,75; p=0,002). y del comportamiento OR 2,11 (IC95\%: Por otro lado, aquellos grupos que tuvie- 1,09 a 4,10; $p=0,025)$. Finalmente los ron una asociación positiva estadística- grupos diagnósticos retraso mental [F70-
F79] con OR 0,14 (IC95\%: 0,03 a 0,61; $\mathrm{p}=0,022)$ y trastornos de conducta y emociones de comienzo en la infancia y adolescencia [F90-F98] OR 0,27 (IC95\%: $0,08$ a 0,91; $p=0,048)$ tuvieron asociación negativa estadísticamente significativa con el grupo etario 12 a 17 años (tabla 3).

Tabla 3. Diagnóstico de las emergencias psiquiátricas en niños y adolescentes según sexo y grupo etario.

\begin{tabular}{|c|c|c|c|c|c|c|c|c|c|c|c|c|c|c|c|c|}
\hline \multirow[b]{3}{*}{ Diagnóstico psiquiátrico (CIE-10)§ } & \multicolumn{8}{|c|}{ Sexo } & \multicolumn{8}{|c|}{ Grupo etario } \\
\hline & \multicolumn{2}{|c|}{$\begin{array}{l}\text { Varón } \\
(\mathrm{n}=114)\end{array}$} & \multicolumn{2}{|c|}{$\begin{array}{c}\text { Mujer } \\
(n=171)\end{array}$} & \multicolumn{4}{|c|}{$\operatorname{IC95\% }$} & \multicolumn{2}{|c|}{$\begin{array}{c}5 \text { a } 11 \text { años } \\
(n=19)\end{array}$} & \multicolumn{2}{|c|}{$\begin{array}{l}12 \text { a } 17 \text { años } \\
(n=266)\end{array}$} & \multicolumn{4}{|c|}{ IC95\% } \\
\hline & $\mathrm{n}$ & $\%$ & $\mathrm{n}$ & $\%$ & OR $\ddagger$ & LI & LS & Valor $p$ & $\mathrm{n}$ & $\%$ & $\mathrm{n}$ & $\%$ & ORT & LI & LS & Valor $\mathrm{p}$ \\
\hline $\begin{array}{l}\text { TM* orgánicos, incluidos los } \\
\text { sintomáticos (F00-F09) }\end{array}$ & 7 & 6,1 & 15 & 8,8 & 1,47 & 0,58 & 3,73 & 0,415 & 1 & 5,3 & 21 & 7,9 & 1,54 & 0,20 & 12,13 & 1 \\
\hline $\begin{array}{l}\text { TM y del comportamiento por } \\
\text { sustancias psicoactivas (F10-F19) }\end{array}$ & 12 & 10,5 & 3 & 1,8 & 0,15 & 0,04 & 0,55 & 0,001 & 0 & 0,0 & 15 & 5,6 & $\mathrm{NC}$ & & & 0,609 \\
\hline $\begin{array}{l}\text { Esquizofrenia, trastornos: esquizotípico } \\
\text { y de ideas delirantes (F20-F29) }\end{array}$ & 48 & 42,1 & 42 & 24,6 & 0,45 & 0,27 & 0,75 & 0,002 & 4 & 21,1 & 86 & 32,3 & 1,79 & 0,58 & 5,56 & 0,307 \\
\hline Trastornos del humor (afectivos) (F30-F39) & 19 & 16,7 & 54 & 31,6 & 2,31 & 1,28 & 4,16 & 0,005 & 6 & 31,6 & 67 & 25,2 & 0,73 & 0,27 & 2.00 & 0,588 \\
\hline $\begin{array}{l}\text { Trastornos neuróticos, por situaciones } \\
\text { estresantes y somatomorfos (F40-F48) }\end{array}$ & 17 & 14,9 & 17 & 9,9 & 0,63 & 0,31 & 1,29 & 0,205 & 2 & 10,5 & 32 & 12,0 & 1,16 & 0,26 & 5,27 & 1 \\
\hline $\begin{array}{l}\text { TC† asociados a disfunciones fisiológicas } \\
\text { y factores somáticos (F50-F59) }\end{array}$ & 1 & 0,9 & 7 & 4,1 & 4,82 & 0,59 & 39,74 & 0,107 & 0 & 0,0 & 8 & 3,0 & $\mathrm{NC}$ & & & 1 \\
\hline $\begin{array}{l}\text { Trastornos de la personalidad y del } \\
\text { comportamiento del adulto (F60-F69) }\end{array}$ & 14 & 12,3 & 39 & 22,8 & 2,11 & 1,09 & 4,10 & 0,025 & 1 & 5,3 & 52 & 19,5 & 4,37 & 0,57 & 33,52 & 1 \\
\hline Retraso mental (F70-F79) & 6 & 5,3 & 4 & 2,3 & 0,43 & 0,12 & 1,56 & 0,133 & 3 & 15,8 & 7 & 2,6 & 0,14 & 0,03 & 0,61 & 0,022 \\
\hline $\begin{array}{l}\text { Trastornos del desarrollo psicológico } \\
\text { (F80-F89) }\end{array}$ & 2 & 1,8 & 0 & 0,0 & $\mathrm{NC}$ & & & 0,159 & 1 & 5,3 & 1 & 0,4 & 0,07 & 0,01 & 1,13 & 0,129 \\
\hline $\begin{array}{l}\text { TC y de emociones de comienzo en la } \\
\text { infancia y adolescencia (F90-F98) }\end{array}$ & 8 & 7,0 & 14 & 8,2 & 1,18 & 0,48 & 2,92 & 0,717 & 4 & 21,1 & 18 & 6,8 & 0,27 & 0,08 & 0,91 & 0,048 \\
\hline Otros (Z00-99 y G40) & 1 & 0,9 & 4 & 2,3 & 2,70 & 0,30 & 24,53 & 0,651 & 1 & 5,3 & 4 & 1,5 & 0,28 & 0,03 & 2,59 & 0,294 \\
\hline
\end{tabular}

${ }^{*} \mathrm{TM}=$ Trastornos mentales $\uparrow \mathrm{TC}=$ Trastornos del comportamiento, $\neq$ Condición de riesgo: sexo mujer

ๆCondición de riesgo: grupo etario 12 a 17 años. §Más de una alternativa posible. 
Tabla 4. Variables clínicas de emergencias psiquiátricas en niños y adolescentes según sexo.

\begin{tabular}{|c|c|c|c|c|c|c|c|c|}
\hline \multirow{2}{*}{ Variables } & \multicolumn{2}{|c|}{ Varón $(n=114)$} & \multicolumn{2}{|c|}{ Mujer $(n=171)$} & \multicolumn{4}{|c|}{ IC95\% } \\
\hline & $\mathrm{n}$ & $\%$ & $\mathrm{n}$ & $\%$ & ORt & LI & LS & Valor $p$ \\
\hline \multicolumn{9}{|l|}{ Tratamiento* } \\
\hline Antipsicótico & 83 & 72,8 & 109 & 63,7 & 0,66 & 0,39 & 1,10 & 0,110 \\
\hline Antidepresivo & 25 & 21,9 & 49 & 28,7 & 1,43 & 0,82 & 2,49 & 0,205 \\
\hline Estabilizadores del ánimo & 26 & 22,8 & 30 & 17,5 & 0,72 & 0,40 & 1,30 & 0,273 \\
\hline Benzodiazepinas & 78 & 68,4 & 124 & 72,5 & 1,22 & 0,73 & 2,05 & 0,456 \\
\hline Psicoterapia & 2 & 1,8 & 1 & 0,6 & 0,33 & 0,03 & 3,68 & 0,566 \\
\hline Otros & 6 & 5,3 & 5 & 2,9 & 0,54 & 0,16 & 1,82 & 0,357 \\
\hline Ninguno & 1 & 0,9 & 4 & 2,3 & 2,71 & 0,30 & 24,53 & 0,651 \\
\hline \multicolumn{9}{|c|}{ Horario de atención } \\
\hline 08:00-19:59 h & 103 & 90,4 & 163 & 95,3 & 2,18 & 0,85 & 5,59 & 0.099 \\
\hline 20:00-07:59 h & 11 & 9,6 & 8 & 4,7 & 1 & & & \\
\hline \multicolumn{9}{|c|}{ Destino de derivación } \\
\hline Sala de observación & 55 & 48,2 & 86 & 50,3 & 1,09 & 0,68 & 1,74 & 0,735 \\
\hline Consulta externa & 59 & 51,8 & 85 & 49,7 & 1 & & & \\
\hline
\end{tabular}

* Más de una alternativa posible, †Condición de riesgo: sexo mujer.

Tabla 5. Variables clínicas de emergencias psiquiátricas en niños y adolescentes según grupo etario

\begin{tabular}{|c|c|c|c|c|c|c|c|c|}
\hline \multirow[t]{2}{*}{ Variables } & \multicolumn{2}{|c|}{5 a 11 años $(n=19)$} & \multicolumn{2}{|c|}{$\begin{array}{c}12 \text { a } 17 \text { años } \\
(\mathrm{n}=266)\end{array}$} & \multicolumn{4}{|c|}{ IC95\% } \\
\hline & $\mathrm{n}$ & $\%$ & $\mathrm{n}$ & $\%$ & $\mathrm{OR} \dagger$ & LI & LS & Valor $p$ \\
\hline \multicolumn{9}{|l|}{ Tratamiento* } \\
\hline Antipsicótico & 11 & 57,9 & 181 & 68,0 & 1,55 & 0,60 & 3,99 & 0,448 \\
\hline Antidepresivo & 5 & 26,3 & 69 & 25,9 & 0,98 & 0,34 & 2,82 & 1 \\
\hline Estabilizadores del ánimo & 6 & 31,6 & 50 & 18,8 & 0,50 & 0,18 & 1,38 & 0,227 \\
\hline Benzodiazepinas & 6 & 31,6 & 196 & 73,7 & 6,07 & 2,22 & 16,58 & $<0,001$ \\
\hline Psicoterapia & 2 & 10,5 & 1 & 0,4 & 0,03 & 0,01 & 0,37 & 0,012 \\
\hline Otros & 1 & 5,3 & 10 & 3,8 & 0,70 & 0,09 & 5,80 & 0,538 \\
\hline Ninguno & 0 & 0,0 & 5 & 1,9 & NC & & & 1 \\
\hline \multicolumn{9}{|l|}{ Horario de atención } \\
\hline 08:00-19:59 h & 18 & 94,7 & 248 & 93,2 & 0,77 & 0,10 & 6,06 & 1 \\
\hline 20:00-07:59 h & 1 & 5,3 & 18 & 6,8 & 1 & & & \\
\hline \multicolumn{9}{|l|}{ Destino de derivación } \\
\hline Sala de observación & 0 & 0,0 & 141 & 53,0 & NC & & & $<0,001$ \\
\hline Consulta externa & 19 & 100 & 125 & 47,0 & & & & \\
\hline
\end{tabular}

* Más de una alternativa posible, † Condición de riesgo: grupo etario 12-17 años. 
Los tratamientos más utilizados fueron las benzodiazepinas y antipsicóticos en $202(70,9 \%)$ y $192(67,4 \%)$ pacientes, respectivamente. El uso de benzodiazepinas se relacionó positivamente con el grupo etario 12 a 17 años, con OR 6,07 (IC95\%: 2,22 a 16,58; $p<0,001$ ) (tablas 4 y $5)$. Hubo mayor frecuencia de emergencias en el horario diurno (08:00 a 19:59 h) en 266 (93,3\%) pacientes. El 49\% necesitó internamiento en sala de observación.

\section{DISCUSIÓN}

Los resultados del presente estudio evidenciaron que las atenciones pediátricas de emergencia constituyeron más del $10 \%$ del total de atenciones en un hospital psiquiátrico nacional. Se evidenció diferencia significativa en cuanto a sexo, siendo más frecuente en mujeres. Existió un incremento de su prevalencia en concordancia con la literatura ${ }^{(9)}$, ya que fue casi el doble a comparación con reportes del año 2003 $(n=136)$ y $2004 \quad(n=145)$, y ligeramente mayor desde el $2005(n=251)^{(10,11)}$.

La edad media fue menor en comparación a los años anteriores, apreciándose su descenso desde 15,9 años ( $D E=1,21$ ) en $2003,15,8$ años ( $D E=1,1)$ en 2004 y 15,7 años ( $D E=1,29)$ en 2005; hasta 14,8 años ( $D E=2.07$ ) en los datos presentados en este estudio (2014). Sin embargo, estudios internacionales difieren de estos resultados, habiendo encontrado una edad media de 9,89 años (12). Al respecto, el haberse presentado con mayor frecuencia a edades tempranas, podría obedecer a diversos factores de riesgo relacionados a cambios sociales y familiares en la sociedad a lo largo de estos años ${ }^{(9,13,14)}$ (violencia doméstica, abandono parental y abuso físico, psicológico, doméstico y escolar, entre otros) los cuales serían necesarios esclarecer en futuros estudios. El rango de edad de 12 a 17 años no presentó variación en la prevalencia en comparación a estudios pasados. Se atendió a una mayor población de Lima Este. Sin embargo, cabe resaltar que hasta un $27 \%$ acudieron de otras áreas de Lima a pesar de políticas de sectorización en la atención de salud.
No existen estudios nacionales previos que especifiquen los motivos concretos por los cuales los niños y adolescentes acuden a emergencia pediátrica. Este estudio contribuyó en explorar características locales, corroborando que, en concordancia con reportes internacionales, la heteroagresividad fue la principal causa de admisión ${ }^{(15,16)}$. Sin embargo, la presentación de síntomas psicóticos e irritabilidad fueron casi tan frecuentes como la conducta suicida. Probablemente, este hecho estuvo relacionado a la mayor frecuencia de presentación de trastornos psicóticos por emergencia que, por su fenomenología, son motivo de desconcierto y consulta inmediata por parte de la familia.

Con respecto a la conducta suicida, el presente estudio evidenció que las mujeres tuvieron hasta tres veces más riesgo de presentar ideación suicida. Con respecto al intento suicida, no se hallaron datos estadísticamente significativos asociados al sexo, como los reportados en otros estudios ${ }^{(17,18)}$.

Los trastornos psicóticos y del ánimo estuvieron más frecuentemente asociados a emergencias pediátricas infantiles a nivel local, en concordancia con estudios anteriores ${ }^{(10,11)}$. Al respecto, los datos de este estudio indicaron que el sexo femenino tuvo hasta dos veces más riesgo de presentar algún trastorno del ánimo. Sin embargo, estos datos contrastaron con los reportados a nivel internacional, en donde los trastornos de conducta y trastornos de adaptación fueron los más frecuentes ${ }^{(18)}$.

Un hallazgo particular del presente estudio fue que los trastornos de personalidad escalaron en el ranking de presentación como diagnóstico asociado a una emergencia psiquiátrica en la adolescencia, a pesar de ser diagnósticos establecidos para la población adulta, teniendo el sexo femenino hasta dos veces más riesgo de mostrarlo. El Manual Diagnóstico y Estadístico de los Trastornos Mentales (DSM-5) ha presentado nuevas alternativas para el abordaje diagnóstico de personalidad, y aun se establece que las características de los mismos pueden aparecer desde la adolescencia o inicios de la edad adulta. El trastorno de ines- tabilidad emocional de la personalidad (F60.3), fue más frecuente probablemente porque, en la actualidad, existe mayor literatura que evidencia la validez de su diagnóstico entre los adolescentes, los cuales pueden beneficiarse de la intervención temprana, siempre y cuando esté presente un cuadro característico y sólido que cumpla la mayoría de criterios ${ }^{(19)}$. Sin embargo, la atención de emergencia en el HHV estuvo a cargo de psiquiatras no especialistas en población pediátrica, por lo que los criterios clínicos pudieron no ser uniformes. Por ello, se recomienda realizar estudios en los que la atención se encuentre a cargo de expertos en esta población.

Con respecto a trastornos asociados a consumo de sustancias, el sexo femenino tuvo $85 \%$ menos riesgo. Al respecto, estudios nacionales (20) informaron una diferencia clara de género, siendo más frecuente el uso de sustancias ilegales en varones (marihuana, cocaína, otros), cierta similitud en sustancias legales en ambos sexos (alcohol, tabaco, otros) y mayor consumo de drogas médicas en mujeres (benzodiazepinas, opioides). Por lo tanto, se considera que, en este punto, los datos del presente estudio no fueron concluyentes, toda vez que en muchas ocasiones no se encontraron registros completos de antecedentes de consumo de sustancias (sobretodo drogas médicas y legales). Se necesitan estudios que puedan esclarecer estos hallazgos.

El horario diurno presentó casi la totalidad de la demanda de atención de emergencia, a diferencia de otros estudios que remarcaban el horario nocturno (21). La mitad de las atenciones necesitó internamiento en sala de observación, siendo incluso mayor en comparación a reportes internacionales ${ }^{(22)}$.

Es importante considerar la necesidad de estandarizar criterios básicos prehospitalarios que determinen una correcta evaluación psiquiátrica pediátrica (estado mental alterado, ingestión autolesiva de venenos, lesión traumática, queja médica no relacionada y violación) con la finalidad de generar ahorro en costos de admisión a un centro hospitalario ${ }^{(23)}$. Sin embargo, actualmente en Perú no se cuenta con profesionales especialistas 
en psiquiatría infantil de guardia, y los internamientos aún se realizan en salas de adultos.

Finalmente, ante esta problemática, la prevención y una adecuada red de servicios de referencia parecen ser una de las estrategias con mayor aceptación a nivel internacional y que muestra resultados alentadores. Se ha demostrado que intervenciones psicosociales tempranas a fin de determinar riesgos potenciales pueden reducir el riesgo de admisiones a emergencia por violencia, autoagresión o problemas de alcohol y drogas ${ }^{(24)}$. Además, en un estudio se observó que los adolescentes dados de alta del servicio de urgencias sin ingreso, mantuvieron un mayor riesgo de suicidio. Esto subraya la importancia de las intervenciones a largo plazo, incluso en adolescentes cuya presentación inicial no era de manera suficiente grave como para justificar la admisión ${ }^{(25)}$. Por esta razón, se ha observado que los aspectos del cuidado de la salud mental que requieren una mayor y urgente investigación son el tipo y la calidad en el manejo de la atención a los niños y adolescentes. El objetivo es mejorar parámetros y estrategias de la práctica médica ${ }^{(26) .}$

Una de las principales limitaciones de este estudio fue la calidad de registro en la historia clínica de emergencia, siendo en muchos casos escueta y limitada en la exploración de antecedentes. Además, todas las evaluaciones fueron realizadas por psiquiatra general 0 médico residente, ya que no se cuenta con especialista en Psiquiatría Infantil de guardia.

Concluyendo, las emergencias psiquiátricas pediátricas presentaron mayor prevalencia y edad media más temprana en relación a años anteriores. Ocurrieron más en el sexo femenino y en la adolescencia. El motivo más frecuente de consulta fue la heteroagresividad. Las alucinaciones e irritabilidad tuvieron similar frecuencia como la conducta suicida. Los trastornos mentales asociados más frecuentes fueron los psicóticos, del ánimo y personalidad. Las mujeres tienen un mayor riesgo de tener ideación suicida, trastorno de ánimo y trastorno de personalidad. Las benzodiazepinas y antipsicóticos son los fármacos más utilizados. La mayoría de atenciones se produjo en el horario diurno y la mitad necesitó hospitalización. Se requiere criterios estandarizados y guías de práctica clínicas, así como contar con especialistas en Psiquiatría Infantil de guardia (diurna) e instalación de ambientes diferenciados.

\section{AGRADECIMIENTOS}

A todo el personal del Departamento de Emergencia y el Departamento de Estadística del Hospital Hermilio Valdizán, por las facilidades brindadas para la realización de este trabajo.

\section{REFERENCIAS BIBLIOGRÁFICAS}

1. Hawton K, Saunders KE, O'Connor RCl. Selfharm and suicide in adolescents. Lancet. 2012 Jun;379(9834):2373-82. DOI: 10.1016/S01406736(12)60322-5

2. Hawton K, Bergen H, Kapur N, Cooper J, Steeg S, Ness J, et al. Repetition of self-harm and suicide following self-harm in children and adolescents: findings from the Multicentre Study of Self-harm in England. J Child Psychol Psychiatry. 2012 Dec;53(12):1212-9. DOI: 10.1111/j.14697610.2012.02559.x

3. Viner RM, Ozer EM, Denny S, Marmot M, Resnick $M$, Fatusi A, et al. Adolescence and the social determinants of health. Lancet Lond Engl. 2012 Apr 28;379(9826):1641-52. DOI: 10.1016/S01406736(12)60149-4.

4. Pumariega AJ, Rothe E. Cultural considerations in child and adolescent psychiatric emergencies and crises. Child Adolesc Psychiatr Clin N Am 2003 Oct;12(4):723-24,vii. DOI: 10.1016/S10564993(03)00038-5

5. Berthaut E, Marcelli D. [Psychiatric emergencies in adolescents]. Rev Prat. 2003 Jun 1;53(11):1191-6.

6. Clark C, Rodgers B, Caldwell T, Power C, Stansfeld S. Childhood and adulthood psychological ill health as predictors of midlife affective and anxiety disorders: the 1958 British Birth Cohort. Arch Gen Psychiatry. 2007 Jun;64(6):668-78. DOI: 10.1001/ archpsyc.64.6.668

7. National Research Council (US) and Institute of Medicine (US) Committee on the Prevention of Mental Disorders and Substance Abuse Among Children, Youth, and Young Adults: Research Advances and Promising Interventions. Preventing Mental, Emotional, and Behavioral Disorders Among Young People: Progress and Possibilities [Internet]. Recuperado el 2016. Disponible de: http://www.ncbi.nlm.nih.gov/books/NBK32775/

8. Sangermani R. Problemi di salute mentale nell'infanzia e nell'adolescenza: criticità nella pratica e nella modalità di intervento. Quaderni Acp. 2014;21(5):210-3
9. Herbert A, Gilbert R, González-Izquierdo A, Li L. Violence, self-harm and drug or alcohol misuse in adolescents admitted to hospitals in England for injury: a retrospective cohort study. BMJ Open 2015 Feb;5(2):e006079. DOI: 10.1136/bmjopen-2014-006079.

10. Toledo Castillo M. Atención de adolescentes en los servicios de Salud Mental del Hospital Hermilio Valdizán. Avances en Salud Mental Relacional. 2006 Jul;5(2):1-37.

11. Toledo M. Variables clínicas y demográficas de las urgencias psiquiátricas infantiles en el Hospital Hermilio Valdizán. Rev Psiq Salud Mental Hermilio Valdizán. 2004 Jun;5(1):83-91.

12. Encarnação R, Moura M, Gomes F, Da Silva PC. [Characterization of the cases referred and consulted in a child and adolescent psyschiatry clinic. A retrospective study]. Acta Médica Port. 2011 Dec;24(6):925-34.

13. Pottick KJ, McAlpine DD, Andelman RB. Changing patterns of psychiatric inpatient care for children and adolescents in general hospitals, 1988-1995. Am J Psychiatry. 2000 Aug;157(8):1267-73. DOI: 10.1176/appi.ajp.157.8.1267

14. Merikangas KR, He J-P, Burstein M, Swanson SA, Avenevoli S, Cui L, et al. Lifetime prevalence of mental disorders in U.S. adolescents: results from the National Comorbidity Survey Replication-Adolescent Supplement (NCS-A). J Am Acad Child Adolesc Psychiatry. 2010 Oct;49(10):980-9. DOI: 10.1016/j.jaac.2010.05.017.

15. Chun TH, Katz ER, Duffy SJ, Gerson RS. Challenges of managing pediatric mental health crises in the emergency department. Child Adolesc Psychiatr Clin N Am. 2015 Jan;24(1):21-40. DOI: 10.1016/j.chc.2014.09.003.

16. Knott JC, Bennett D, Rawet J, Taylor DM. Epidemiology of unarmed threats in the emergency department. Emerg Med Australas EMA. 2005 Aug;17(4):351-8. DOI: 10.1111/j.17426723.2005.00756.x

17. Eaton DK, Kann L, Kinchen S, Shanklin S, Flint KH, Hawkins J, et al. Youth risk behavior surveillance United States, 2011. Morb Mortal Wkly Rep Surveill Summ Wash DC 2002. 2012 Jun 8;61(4):1-162.

18. Di Lorenzo R, Cimino N, Di Pietro E, Pollutri G, Neviani V, Ferri P. A 5-year retrospective study of demographic, anamnestic, and clinical factors related to psychiatric hospitalizations of adolescent patients. Neuropsychiatr Dis Treat. 2016;12:191201. DOI: $10.2147 /$ NDT.S93874.

19. Kaess M, Brunner R, Chanen A. Borderline personality disorder in adolescence. Pediatrics. 2014 Oct;134(4):782-93. DOI: 10.1542/peds.2013-3677.

20. DEVIDA. I Estudio sobre prevención y consumo de drogas en la población general de Lima Metropolitana y el Callao - 2013[Internet]. Recuperado el 2016. Disponible de: http://www.devida.gob.pe/ wp-content/uploads/2015/02/I_Estudio_Lima_Callao_completo.pdf

21. Case SD, Case BG, Olfson M, Linakis JG, Laska EM. Length of stay of pediatric mental health emergency department visits in the United States. J Am Acad Child Adolesc Psychiatry. 2011 Nov;50(11):1110-9. DOI:10.1016/j. jaac.2011.08.011.

22. Bell L, Stargatt R, Bosanac P, Castle D, Braitberg G, Coventry N. Child and adolescent mental health problems and substance use presentations to an emergency department. Australas Psychiatry Bull 
R Aust N Z Coll Psychiatr. 2011 Dec;19(6):521-5 DOI: 10.3109/10398562.2011.603329.

23. Santillanes G, Donofrio JJ, Lam CN, Claudius I. Is medical clearance necessary for pediatric psychiatric patients?. J Emerg Med. 2014 Jun;46(6):800-7. DOI: 10.1016/j.jemermed.2013.12.003.

24. Asarnow, Baraff L, Berk M, Grob C, Devich-Navarro M, Suddath R, et al. Effects of an emergency department mental health intervention for linking pediatric suicidal patients to follow-up mental health treatment: a randomized controlled trial. Psychiatr Serv Wash DC. 2011 Nov 1;62(11):1303-9. DOI: 10.1176/appi.ps.62.11.1303

25. Finkelstein $Y$, Macdonald EM, Hollands S, Hutson JR, Sivilotti MLA, Mamdani MM, et al. Long-term outcomes following self-poisoning in adolescents: a population-based cohort study. Lancet Psychiatry. 2015 Jun;2(6):532-9. DOI: 10.1016/ S2215-0366(15)00170-4.
26. Newton AS, Rosychuk RJ, Niu X, Radomski AD, McGrath PJ. Predicting time to emergency department return for anxiety disorders and acute stress reactions in children and adolescents: a cohort study. Soc Psychiatry Psychiatr Epidemiol. 2015 Aug;50(8):1199-206. DOI: 10.1007/s00127015-1073-7. 